\title{
Luminous Infrared Sources in the Local Group: Identifying the Missing Links in Massive Star Evolution
}

\author{
N. Britavskiy ${ }^{1}$, A. Z. Bonanos ${ }^{1}$ and A. Mehner ${ }^{2}$ \\ ${ }^{1}$ IAASARS, National Observatory of Athens, Greece \\ email: britvavskiy@gmail.com \\ ${ }^{2}$ ESO, Santiago, Chile
}

\begin{abstract}
We present the first systematic survey of dusty massive stars (RSGs, LBVs, sgB[e]) in nearby galaxies, with the goal of understanding their importance in massive star evolution. Using the fact that these stars are bright in mid-infrared colors due to dust, we provide a technique for selecting and identifying dusty evolved stars based on the results of Bonanos et al. (2009, 2010), Britavskiy et al. (2014), and archival Spitzer/IRAC photometry. We present the results of our spectroscopic follow-up of luminous infrared sources in the Local Group dwarf irregular galaxies: Pegasus, Phoenix, Sextans A and WLM. The survey aims to complete the census of dusty massive stars in the Local Group.
\end{abstract}

Keywords. stars: emission-line, supergiants, galaxies: individual (Pegasus, Phoenix, Sextans A, WLM): local group

\section{Introduction}

The role of episodic mass loss in massive star evolution is one of the most important open questions of current stellar evolution theory (Smith 2014). Episodic mass loss produces dust and therefore causes evolved massive stars to be very luminous in the midinfrared and dim at optical wavelengths. We aim to increase the number of investigated luminous mid-IR sources to shed light on the late stages of these objects. To achieve this we employed mid-IR selection criteria to identify dusty evolved massive stars in 4 nearby galaxies.

We selected 4 star-forming dwarf irregular galaxies (dIrr) with existing Spitzer photometry, namely Pegasus, Phoenix, Sextans A, and WLM. This work is based on the ESO/VLT proposal 090.D-009 where we observed 99 dusty massive star candidates with the FORS2 MXU spectrograph. The candidate selection was based on mid-IR colors, using $3.6 \mu \mathrm{m}$ and $4.5 \mu \mathrm{m}$ photometry from archival Spitzer Space Telescope images of nearby galaxies and $J$-band photometry from 2MASS. The targets selection technique was based on the results of Bonanos et al. (2009, 2010), which illustrated that each type of dusty massive stars occupies its own region on the color magnitude diagram (CMD). The most luminous targets in mid-IR colors $(M[3.6]<-9$ mag) were observed in $4 \mathrm{dIrr}$ galaxies with exposure time $600 \mathrm{~s}$, a spectral resolution of $\mathrm{R} \approx 440$ and $\mathrm{S} / \mathrm{N} \approx 30$.

\section{Preliminary results}

The spectral type classification revealed 8 new red supergiants (RSGs), 3 new luminous blue variable $(\mathrm{LBV}) / \mathrm{B}[\mathrm{e}]$ supergiant $(\mathrm{sgB}[\mathrm{e}])$ candidates, and independently re-identified 3 RSGs in Sextans A (Britavskiy et al. 2014) and 4 RSGs in WLM (Levesque \& Massey 


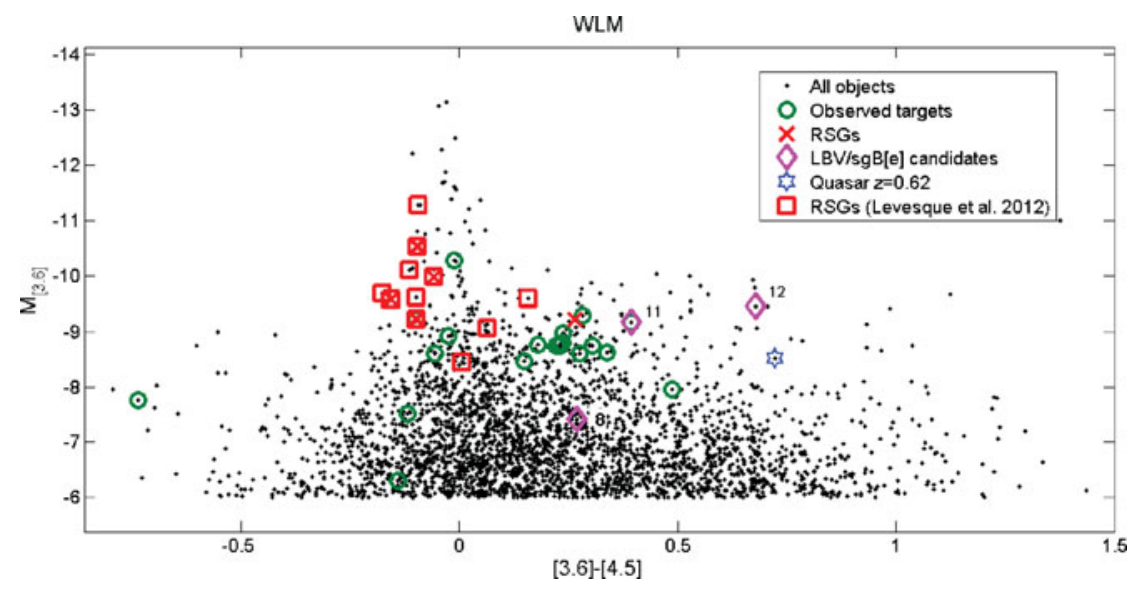

Figure 1. [3.6]-[4.5] color magnitude diagram for one of the observed dwarf irregular galaxies WLM. The positions of our investigated targets are labeled using different symbols. The numbers indicate the emission line objects.

2012). The positions of observed targets on the infrared CMD for WLM are presented in Figure 1.

The most numerous massive dusty objects that we have identified are the RSGs. We have found: 2 new RSGs in Pegasus; 1 in Phoenix; 7 in Sextans A, 3 of them have been independently previously discovered by Britavskiy et al. (2014). Also, we found 5 new RSGs in WLM, 4 of them have been identified by Levesque \& Massey (2012). The newly discovered RSGs belong to the late spectral types (typically K-M). The positions on the color magnitude diagram of newly discovered and known RSGs for WLM are presented in Figure 1. The region of RSGs is well visible on this diagram, with [3.6] $-[4.5]<0$. For RSGs for which we have 2 independent observations we will perform spectral type comparison for investigation of the spectral type variability as described in Levesque (2010) and Levesque \& Massey (2012). Furthermore, the sample of 4 dIrr galaxies provide to us a good opportunity to investigate dependencies between average spectral types of RSGs vs. metallicities of galaxies. Among the reduced spectra we have identified three emission line spectra. The spectra have prominent hydrogen and iron emission lines that give a hint about the spectral type of these objects, namely B type. Preliminary analysis of the spectra and available photometry suggest the following spectral classification for the objects, which are numbered according to their aperture number on the slit: WLM 8 an LBV candidate with an HII region, WLM 11 - an LBV candidate, WLM 12 - a sgB[e] candidate. Note, there are no He emission lines, that are typical for LBVs. Massey et al. (2007) previously reported that WLM 8 and WLM 12 are hydrogen emission objects.

\section{References}

Bonanos, A. Z., Lennon, D. J., Köhlinger, F., et al. 2010, AJ, 140, 416

Bonanos, A. Z., Massa, D. L., Sewilo, M., et al. 2009, AJ, 138, 1003

Britavskiy, N. E., Bonanos, A. Z., Mehner, A., et al. 2014, A\& A, 562, A75

Levesque, E. M. 2010, Vol. 425 of Astronomical Society of the Pacific Conference Series, p. 103 Levesque, E. M. \& Massey, P. 2012, AJ, 144, 2

Massey, P., Olsen, K. A. G., Hodge, P. W., et al. 2007, AJ, 133, 2393

Smith, N. 2014, ARAA in press 